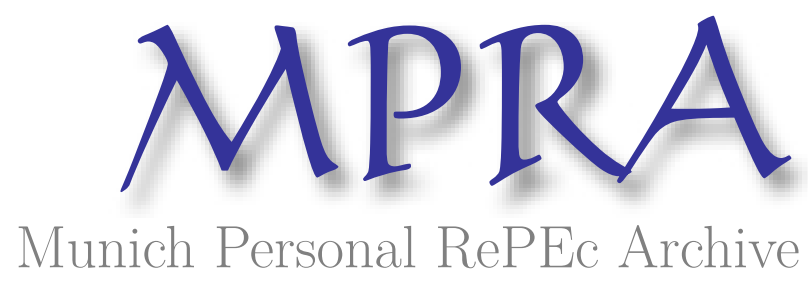

\title{
Topological complexity of manifolds of preferences
}

Chichilnisky, Graciela

1986

Online at https://mpra.ub.uni-muenchen.de/8119/

MPRA Paper No. 8119, posted 07 Apr 2008 01:37 UTC 
REPRINTED FROM:

CONTRIBUTIONS TO

MATHEMATICAL

ECONOMICS

In Honor of Gérard Debreu

Edited by

WERNER HILDENBRAND, University of Bonn

ANDREU MAS-COLELL, Harvard University

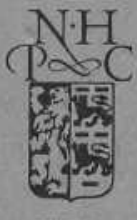

1986

NORTH-HOLLAND

Amsterdam - New York - Oxford - Tokyo 


\section{TOPOLOGICAL COMPLEXITY OF MANIFOLDS OF PREFERENCES*}

\section{GRACIELA CHICHILNISKY}

Columbia University, New York, NY, USA

\section{Introduction}

The problem of endowing preferences with manifold structures emerged from discussions with Gérard Debreu in 1975. Time has shown that such structures can be useful in understanding the behavior of economic systems.

In Chichilnisky (1976) spaces of smooth preferences were endowed with a Hilbert manifold structure, and this was used to study the existence and structural stability of competitive equilibria in economies where preferences might be non-monotonic and non-convex. This paper constructs manifolds of preferences and applies this construction to the aggregation of preferences. We examine the topological complexity of manifolds of smooth preferences and use this to determine when appropriate aggregation rules exist and when they do not.

In mathematical terms, a smooth preference is an oriented foliation of the choice space. General spaces of foliations are still poorly understood. Our approach is to consider subspaces of foliations which are of special interest in economic theory and which can be endowed with manifold structures. These spaces are large enough to be infinite-dimensional, and to include non-convex and non-monotonic preferences. Spaces of convex and monotonic preferences are shown to be submanifolds.

In those cases where our construction succeeds in endowing preference spaces with a manifold structure, it also serves to show that these manifolds are contractible, or topologically trivial. These manifolds therefore satisfy the necessary and sufficient condition of Chichilnisky and Heal (1983) for the existence of aggregation rules which are continuous, anonymous and respect unanimity.

\footnotetext{
* This research was supported by NSF grants No. SES-84-09857 and SES-84-20244. Comments from Andreu Mas-Colell and Dennis Sullivan are gratefully acknowledged.
} 
However, the larger space of all preferences given by regular foliations is shown to be topologically complex: it contains a sphere as a retract. Therefore, this space is not contractible and does not admit continuous and anonymous aggregation rules which respect unanimity.

The next section contains definitions and notation. Section 3 proves that regular preferences can be viewed as retractions of the choice space and that spaces of preferences are two-fold covers of spaces of retractions. Certain spaces of retractions are then endowed with (Hilbert) manifold structures. Section 4 shows that the manifolds in Section 3 are contractible: they therefore admit appropriate aggregation rules and continuous representation into function spaces. It is then shown that the space of all regular preferences is topologically complex: it has a sphere as a retract. Therefore this space does not admit appropriate aggregation, as shown in Chichilnisky (1980).

\section{Notation and definition}

$X$ denotes a choice space, which is the closed unit cube $I^{n}$ or unit ball $B^{n}$ in Euclidean space $R^{n}$, or any manifold with boundary which is $C^{k}$ diffeomorphic to either $I^{n}$ or $B^{n},{ }^{1}(k \geqq 1)$. A function $f: X \rightarrow R$ defines a preference on $X$, by the rule: $x \in X$ is at least as desirable as $y \in X(x p y)$ when $f(x) \geqq f(y)$. If $f$ is continuous, the preference it defines is said to be continuous. A $C^{k}$ function $f: X \rightarrow R$ is regular $(k \geqq 1)$ when its derivative $\mathrm{D} f(x)$ never vanishes in the interior of $X$, and the restriction of $\mathrm{D} f$ on the boundary of $X$, $\partial X$, has only two zeroes and is otherwise transversal to $\partial X$.

A codimension-one globally integrable oriented foliation of $X$ is a function $v$ : $X \rightarrow S^{n-1}$, where $S^{n-1}$ is the $(n-1)$ th sphere, such that there exists a $C^{1}$ map $f: X \rightarrow R$ with

$$
v(x)=\lambda(x) \mathrm{D} f(x),
$$

for all $x$ in $X$, and for some continuous positive map $\lambda: X \rightarrow R$. A leaf of this foliation is a hypersurface of the map $f ; f$ is said to define the foliation.

Many functions $f: X \rightarrow R$ define the same oriented foliation on $X$ : any two $C^{1}$ functions $g, f: X \rightarrow R$ having the same set of hypersurfaces and increasing in the same direction, define the same oriented foliation on $X$. Any two such functions will also define the same preference on $X$.!

\footnotetext{
${ }^{1} C^{k}$ indicates $k$ times continuously differentiable. For a definition of a $C^{k}$ manifold, see Abraham and Robbins (1967), and for manifolds with boundary, see Hirsch and Mazur (1974). A $C^{k}$ manifold $X \subset R^{n}$ with boundary is $C^{k}$ diffeomorphic to another $Y \subset R^{m}$ if there exists a one-to-one onto $C^{k}$ map $f: U(X) \rightarrow U(Y)$, with $C^{k}$ inverse $f^{-1}(U(Y)) \rightarrow U(X)$, where $U(X)$ and $U(Y)$ are neighborhoods of $X$ and $Y$ in $R^{n}$ and $R^{m}$, respectively. $f: X \rightarrow R$ is called $C^{k}$ if it admits a $C^{k}$ extension to a neighborhood of $X$ in $R^{n}$.
} 


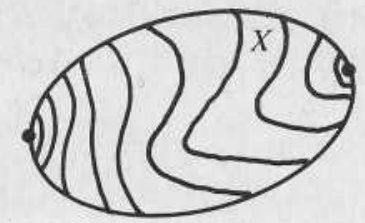

Figure 1. A regular preference on $X$.

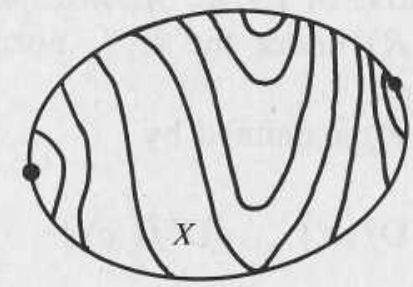

Figure 2. An oriented foliation of $X$ which does not define a regular preference.

The relation between utility functions and preferences is many-to-one, and so is the relation between functions and foliations. However, the correspondence between oriented foliations and preferences is one-to-one. The leaves of the foliation are the indifference surfaces of the preference. ${ }^{2}$

This motivated one of Gerard Debreu's definitions of smooth preferences [Debreu (1972)] as globally integrable foliations: it also motivates the definition we give here.

A $C^{k}(k \geqq 1)$ regular preference $p$ is an oriented foliation of $X$ defined by a $C^{k}$ regular function $f: X \rightarrow R$, and such that the leaves of the foliation define a $C^{k}$ coordinate system for $X{ }^{3}$ An integral curve of this foliation is a map $C:[0,1] \rightarrow X$ satisfying for all $t$ in $[0,1] \dot{C}(t)=v(x)$, where $C(t)=x$.

A space $Y \subset R^{n}$ is called contractible if there is a continuous map $d: Y \times[0,1] \rightarrow Y$ such that $d(y, 0)=y$ for all $y$ in $Y$, and $d(y, 1)=y_{0}$ for some $y_{0}$ in $Y$, all $y$ in $Y$. A retraction from a space $Z$ into another $Y \subset Z$ is a continuous function $r: Z \rightarrow Y$ such that $r / Y=i d_{Y}$.

Let $C^{\infty}(X, R)$ denote the space of all infinitely differentiable functions from $X$ to $R$, and $C^{k}(X, R)$ the space of $k$ times continuously differentiable such

${ }^{2}$ An indifference surface $J$ of a preference $p$ consists of points which are all preferred to each other, i.e., indifferent to each other: $x, y \in J$ iff $x p y$ and $y p x$.

${ }^{3}$ That is, there exists a $C^{k}$ diffeomorphism $g: X \rightarrow R^{n}$ such that each leaf is of the form

$$
g^{-1}\left\{\left(x_{1}, \ldots, x_{n}\right) \in R^{n}: x_{i}=\bar{x}_{i}\right\} \text { for some } \bar{x}_{i} \in R \text {. }
$$


functions, $k \geqq 1$. Both $C^{\infty}(X, R)$ and $C^{k}(X, R)$ are linear spaces, with the addition rule $(f+y)(x)=f(x)+g(x)$. The Sobolev norm $\|\cdot\|_{s}$ is defined on $C^{\infty}(X, R)$ by

$$
\|f\|_{s}=\left(\int_{X_{0} \leqq|k| \leqq s} \sum\left|\mathrm{D}^{k} f(x)\right|^{2} \mathrm{~d} x\right)^{1 / 2},
$$

where $\mathrm{D}^{k}$ is the $k$ th derivative of $f$. The Sobolev space $H^{s}(X, R)$ is defined as the completion of $C^{\infty}(X, R)$ under the $\|\cdot\|_{s}$ norm. $H^{s}(X, R)$ is a Hilbert space.

The $C^{k}$ norm on $C^{k}(X, R)$ is defined by

$$
\|f\|_{k}=\sup _{x \in X}\left\|f(x), \mathrm{D} f(x), \ldots, \mathrm{D}^{k} f(x)\right\| .
$$

Sobolev's theorem establishes that $H^{s}(X, R) \subset C^{k}(X, R)$ for $s \geqq n / 2+k$, and this inclusion is continuous and compact [Sobolev (1963) and Skorohod (1974)]. In the following we assume $s \geqq n / 2+k$, and $k \geqq 1$.

\section{A Hilbert manifold of preferences}

Our first step is to show that regular preferences can be viewed as retractions of the choice space. We then show how spaces of retractions can be endowed with manifold structures.

Lemma 1. Let $p$ be a $C^{k}$ regular preference on $X$. Then we can identify $p$ with a unique retraction from $X$ into a regular $C^{k}$ submanifold $I$ of $X{ }^{4}$

Proof. Let $f: X \rightarrow R$ represent the preference $p$. By regularity of $p, f$ attains one minimum and one maximum on $\partial X$. Call these $x_{1}$ and $x_{2}$. Consider now an integral curve $I:[0,1] \rightarrow X$ of the vector field defined by $\mathrm{D} f$, with $I(0)=x_{1}$. The graph of $I$ is a $C^{k}$ regular submanifold of $X$ by definition of $p$. $I(1)=x_{2}$ by proposition 4 of Chichilnisky (1976, p. 47).

Define now a retraction $r: X \rightarrow I$ as follows: $r(x)=d \in I$ such that $f(x)=f(d)$. The map $r$ is well defined because for any $x \in X$ there exists

\footnotetext{
${ }^{4}$ A submanifold $Y \subset X$ is called neat when its boundary $\partial Y$ is the intersection of $Y$ with the boundary $X$ and $Y$ is transversal to $X$ at $\partial Y$; for a definition of transversality see Abraham and Robbins (1967). A submanifold $Y \subset X$ is called regular if it is contractible, $C^{k}(k \geqq 1)$, neat and compact. See also Hirsch (1976).
} 


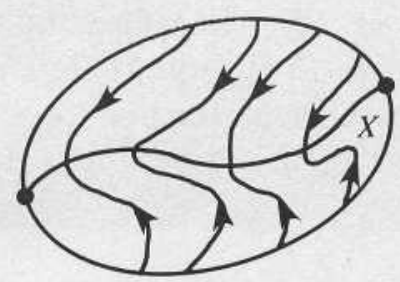

Figure 3. The retraction $r$ is indicated by the arrows. The inverse image under the retraction $r$ of the point $x$ in $I$, is an indifference surface of the preference $p$ defined by $r$.

$d \in I$ with $f(x)=f(d)$ : this follows from connectedness of $I$ and the fact that $f\left(x_{1}\right) \leqq f(x) \leqq f\left(x_{2}\right)$. Furthermore, such a $d$ is unique: by construction of $I$ and the regularity of $p, f$ is strictly increasing along $I$. Continuity of $r$ follows from the smoothness of $p$.

A retraction $r$ from $X$ into a regular $C^{k}$ manifold $I$ of $X$ is called regular if it is a regular map from $X$ to $I$ which is transversal to $\partial X-I=\{x \in \partial X$ : $x \notin I\}$.

Lemma 2. Let I be a regular $C^{k}$ curve ${ }^{5}$ in $X, x \in \partial I$. Any retraction from $X$ to $I$ defines a unique preference $p$ which attains a minimum at $x$. If the retraction is $C^{k}$ and regular, $p$ is $C^{k}$ and regular.

Proof. By the classification theorem for one-dimensional manifolds [Milnor (1965)] $I$ is $C^{k}$ diffeomorphic to $[0,1]$. Let $d: I \rightarrow[0,1]$ be a $C^{k}$ regular diffeomorphism with $d(x)=0$, and $r$ a retraction from $X$ to $I$. Define $f: X \rightarrow R$ as the composition map $d \circ r$. Then $f$ defines a continuous preference $p$ on $X$.

If the retraction $r$ is $C^{k}$, so are $f$ and $p$. To see that $p$ is regular when $r$ is regular, it suffices to recall that for all $x$, the manifold $r^{-1}(x)$ is transversal to $\partial X-I$. It follows that $\partial f / \partial X$ is non-zero everywhere except at two points of $\partial X$.

Note that the above procedure defines the same preference $p$ on $X$, for any regular diffeomorphism $d: I \rightarrow[0,1]$ with $d(x)=0$.

Lemma 3. Let I be a regular $C^{k}$ curve in $X$. Any retraction from $X$ to $I$ defines two continuous preferences on $X$. If the retraction is $C^{k}$ and regular, then the preferences are $C^{k}$ and regular.

${ }^{5} \mathrm{~A} \mathrm{C}^{k}$ regular curve is a one-dimensional regular submanifold of $X$. 
Proof. Let $x \in \partial I$. Then $d \circ r: X \rightarrow R$ defines the same preference on $X$ for all $C^{k}$ regular diffeomorphisms $d: I \rightarrow[0,1]$ satisfying $d(x)=0$. The composition map $d \circ r$ defines another preference for all such $d: I \rightarrow[0,1]$ with $d(x)=1$. Therefore, any retraction from $X$ to $I$ defines exactly two preferences on $X$.

Our next step is to prove that the following diagram exists:

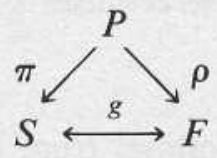

where $P$ is a space of regular preferences, $S$ is a space of regular retractions from $X$ into some curve in $X$, and $F$ is a space of regular foliations on $X$. The maps $\pi$ and $\rho$ are two-fold coverings, and the map $g$ : $S \rightarrow F$ is one-to-one and onto.

Theorem 1. The space $P$ of all $C^{k}$ regular preferences over $X$ can be identified with a two-fold covering ${ }^{6}$ of the space $S$ of all $C^{k}$ regular retractions from $X$ into some $C^{k}$ regular curve of $X$.

Proof. By Lemma 1, any $p \in P$ defines a unique retraction $r$ in $S$ : call this map $\pi: P \rightarrow S$. By Lemma 3 , any $r \in S$ is the image of exactly two preferences in $P$. Therefore, the map $\pi: P \rightarrow S$ is two-to-one.

Let $r \in S$. At each $x \in X$, consider the line generated by the direction orthogonal to the indifference surface of $r$ at $x$. This defines for each $r$ a unique $C^{k}$ map from $X$ to $P^{n}$, the $n$th projective space consisting of lines through the origin in $R^{n}$. Thus we can identify $S$ with a subset of $C^{k}\left(X, P^{n}\right)$ and provide it with the inherited $C^{k}$ topology, $\tau$.

For any $r \in S$ there exists a neighborhood $U_{r}$ in $\tau$ such that $\pi^{-1}\left(U_{r}\right)$ consists of two disjoint sets, $V_{r}$ and $W_{r}$, and by Lemma 2 the restriction maps $\pi / V_{r}$ and $\pi / W_{r}$ are one-to-one. Define on $P$ the topology it inherits from $S$ under this locally invertible map. By construction, $\pi$ is a local homeomorphism. Since $\pi^{-1}(r)$ has exactly two elements for all $r \in S, \pi$ defines a two-fold covering of $S$.

A codimension-one globally integrable foliation of $X$ is a function $v: X \rightarrow P^{n}$, where $P^{n}$ is the $n$th projective space, such that there exists a $C^{1}$ function

${ }^{6} \mathrm{~A}$ two-fold covering or two-fold covering projection $\pi: X \rightarrow Y$ is a continuous map that is a uniform local homeomorphism, and such that for all $y \in Y, \pi^{-1}(y)$ consists exactly of two points [see Spanier (1963, p. 62)]. 
$f: X \rightarrow R$ with

$$
v(x)=\lambda(x) \mathrm{D} f(x),
$$

for all $x$ in $X$, and for some continuous map $\lambda: X \rightarrow R$. The foliation is called $C^{k}$ and regular if its leaves define a $C^{k}$ coordinate system for $X, f$ is regular, and $f$ attains only one maximum and one minimum in $\partial X$. Let $F$ be the space of all $C^{k}$ regular codimension-one globally integrable foliations of $X$.

Theorem 2. There is a one-to-one correspondence between the space of foliations $F$, and the space $S$ of $C^{k}$ regular retractions from $X$ into some $C^{k}$ regular curve of $X$.

Proof. This follows from Lemmas 1 and 2.

Corollary 1. The space of preferences $P$ is a two-fold cover of the space of foliations $F$.

Proof. This follows from Theorems 1 and 2.

Theorem 3. Let I be a $C^{k}$ regular curve in $X$. Then the space of all $C^{k}$ regular retractions from $X$ into $I$ is a Banach manifold. The space of all $H^{S}$ retractions from $X$ into $I$ is a Hilbert manifold consisting of $C^{k}$ maps.

Proof. Let $C^{k}(X, I)$ denote the space of all $C^{k}$ maps from $X$ into $I$ : this is a Banach manifold [see Abraham and Robbins (1967)]. Similarly, let $C^{k}(I, I)$ denote the space of all $C^{k}$ maps from $I$ to $I$, which is also a Banach manifold.

Consider now the map $R: C^{k}(X, I) \rightarrow C^{k}(I, I)$ defined by $R(f)=$ $f / I: I \rightarrow I$. This map is $C^{k}$, and if $i d_{I}: I \rightarrow I$ is the identity map on $I$, then $i d_{I}$ is a regular value of $R$ [see proposition 1 of Chichilnisky (1976)]. Therefore, $R^{-1}\left(i d_{I}\right)$ is a Banach submanifold of $C^{k}(X, I)$ : this is the implicit function theorem on Banach manifolds [see Abraham and Robbins (1967)]. But $R^{-1}\left(i_{I}\right)$ is, by definition, the space of $C^{k}$ retractions from $X$ to $I$. Therefore the space of $C^{k}$ retractions from $X$ to $I$ is a Banach manifold. Since regularity is an open property in $C^{k}(X, I)$, all $C^{k}$ regular retractions are an open subset of a Banach manifold, and thus form a Banach manifold.

Similarly, all $H^{s}$ retractions from $X$ into $I$ are the inverse image in $H^{s}(X, I)$ of the identity map in $H^{s}(I, I)$, under the map $R$. Since the identity map is a regular value of $R$, all $H^{s}$ retractions form a submanifold of the Hilbert manifold $H^{s}(X, I)$. All regular $H^{s}$ retractions from $X$ into $I$ are an open subset of the Hilbert manifold of retractions from $X$ into $I$ : this is 
because regularity is an open property in $C^{k}$, and the inclusion $H^{s} \subset C^{k}$ is a continuous map by Sobolev's theorem [see Chichilnisky (1977b)].

Let $I$ be a regular curve in $X$, given by $I:[0,1] \rightarrow X$.

Corollary 2. The space $P_{I}$ of $C^{k}$ regular preferences on $X$ which attain a maximum at $I(1)$, a minimum of $I(0)$ and are strictly increasing along $I$, can be identified with the Banach manifold of $C^{k}$ regular retraction from $X$ into $I$. The $H^{s}$ regular retractions from $X$ into I define a Hilbert manifold $H_{I}$ of $C^{k}$ regular preferences which are strictly increasing along $I$, and which attain a minimum at $I(0)$.

Proof. The proof of Lemma 1 shows that any $C^{k}$ regular preference which is strictly increasing along $I$ and attains a maximum and minimum at $I(1)$ and $I(0)$ respectively, can be represented by a unique $C^{k}$ regular retraction from $X$ into $I$. Lemma 2 shows that any such retraction defines a unique $C^{k}$ regular preference on $X$. The rest of the corollary follows from Theorem 3 .

Let $X=I^{n}$, the unit cube in $R^{n}$, and $\Delta$ the diagonal in $I^{n}, \Delta=$ $\left\{\left(x_{1}, \ldots, x_{n}\right): x_{i}=x_{j}, \forall i, j\right\} . \Delta$ is a $C^{k}$ regular curve. A preference $p$ on $I^{n}$ is called monotonic if it is represented by a function $f: X \rightarrow R$ satisfying $f(x)>f(y)$ when $x>y$ in the vector order of $R^{n}$. A preference $p$ on $I^{n}$ is called strictly concave when it is represented by a function with a second-order derivative defining a negative definite form.

Corollary 3. The space of $H^{s}$ monotonic preferences on $I^{n}$ is a submanifold of the Hilbert manifold $H_{\Delta}$. The space of $H^{s}$ strictly concave monotonic preferences on $I^{n}$ is a submanifold of $H_{\Delta}$ when $k \geqq 2$.

Proof. The first statement follows from the facts that montonicity is an open property in the $C^{k}$ topology with $k \geqq 1$, and that the inclusion map $H^{s} \subset C^{k}$ is continuous by Sobolev's theorem. The second statement follows from the fact that strict concavity is an open property in the $C^{k}$ topology when $k \geqq 2$.

\section{Topology, aggregation and continuous representation}

Our next step is to show that the manifolds of preferences defined in Section 3 are contractible; they therefore admit appropriate aggregation rules and continuous representation into function spaces [see, e.g., Debreu (1964) and Chichilnisky $(1977 \mathrm{a}, 1981)$. We then show that the larger space of all regular 
preferences is not contractible: it has a sphere as a retract. Therefore this larger space does not admit appropriate aggregation.

Proposition 1. The Hilbert manifold of all $H^{s}$ retractions from $X$ into a $C^{k}$ regular curve $I$ is contractible.

Proof. As in Lemma 2, let $d$ be a $C^{k}$ diffeomorphism from $I$ into $[0,1]$. Let $\bar{f}$ be a retraction in $H^{s}(X, I)$.

Define $\pi: H^{s}(X, I) \times[0,1] \rightarrow H^{s}(X, I)$ by

$$
\pi(f, \lambda)(x)=d^{-1}[\lambda(d(\vec{f}(x)))+(1-\lambda)(d(f(x)))] .
$$

$\pi$ is a continuous map from $H^{s}(X, I) \times[0,1]$ into $H^{s}(X, I)$. For $\lambda=0$, $\pi(f, \lambda)(x)=f(x)$, and for $\lambda=1, \pi(f, 1)(x)=\bar{f}(x)$. Furthermore, for all $\lambda$, $\pi(f, \lambda)$ is a retraction in $H^{s}(X, I)$ when $f$ is a retraction. Therefore, the space of $H^{s}$ retractions from $X$ into $I$ is contractible.

Corollary 4. The Hilbert manifold of preferences $H_{I}$ is contractible. In particular, for any $m \geqq 2$, there exists a continuous aggregation map

$$
\phi:\left(H_{I}\right)^{m} \rightarrow H_{I},
$$

which respects unanimity and anonymity.

Proof. A necessary and sufficient condition for the existence of a continuous anonymous map $\phi:(M)^{k} \rightarrow M$ respecting unanimity is contractibility of $M$, when $M$ is a CW complex [see Chichilnisky and Heal (1983)]. By Proposition 1 and Corollary 2, $H_{I}$ is contractible. However, we have not shown here that $H_{I}$ is a CW complex. We shall therefore offer an alternative proof, which consists of constructing one map $\phi:\left(H_{I}\right)^{m} \rightarrow H_{I}$ with all the desired properties.

Let $d$ be a $C^{k}$ diffeomorphism $d: I \rightarrow[0,1]$ as in Lemma 2. Define $\phi:$ $\left(H_{I}\right)^{m} \rightarrow H_{I}$ by

$$
\phi\left(p_{1}, \ldots, p_{m}\right)(x)=d^{-1}\left(\sum_{i} \frac{1}{m} d\left(p_{i}(x)\right)\right) ;
$$

see Figure 4. $\phi$ is continuous, $\phi(p, \ldots, p)=p$ for all $p$, and $\phi$ is invariant under permutations of the $p_{i}$ 's.

${ }^{7} \phi: Y^{m} \rightarrow Y$ respects anonymity if it is invariant under permutations of its arguments; $\phi$ respects unanimity if it is the identity on the "diagonal" of $Y$, i.e., $\phi(y, \ldots, y)=y, \forall y \in Y$. 


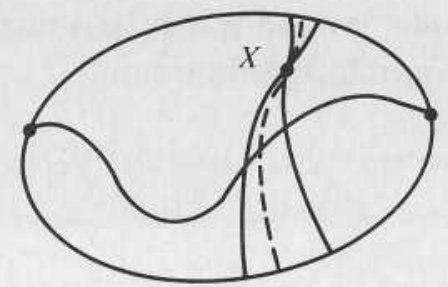

Figure 4. A continuous map $\phi:\left(H_{l}\right)^{2} \rightarrow H_{l}$ which respects anonymity and unanimity. The broken line represents the retraction given by the composition map $d^{-1}\left(\frac{1}{2}\left(d \circ p_{1}+d \circ p_{2}\right)\right.$.

Corollary 5. There exists a continuous representation of the space $H_{I}$ into the space of $H^{s}$ utility functions over the choice space $X, H^{s}(X, R)$.

Proof. For any $p \in H_{I}$, the composition mapping $d \circ p: X \rightarrow R$ defines a continuous representation from $H_{I}$ into $H^{s}(X, R)$, where $d$ is a $C^{k}$ diffeomorphism between $I$ and $[0,1]$ as in Lemma 2.

Theorem 4. The space $P$ of all $C^{k}$ regular preferences on $X$ has as a retract a space diffeomorphic to the $n$th sphere $S^{n}$, where $n$ is the dimension of the choice space $X$.

Proof. Consider first the case $X=B^{n}$. The space $P$ of $C^{k}$ regular preferences on $X$ is a subspace of the space $C^{k}\left(X, S^{n}\right)$; endow this latter space with the $C^{k}$ topology. For any $p \in P$, let $x(p)$ be the minimum of $p$ over $X$. The map $p \rightarrow x(p)$ is continuous, and it maps $P$ into $S^{n}$. Denote this by $\psi: P \rightarrow S^{n}$.

Now, for any point $y \in S^{n}$, let $p(y)$ be the preference having as indifference surfaces hyperplanes which are orthogonal to the segment $[y,-y]$, and with $y$ as a minimum. This defines a continuous one-to-one map $X: S^{n} \rightarrow P$; let its image in $P$ be denoted also $S^{n}$. Since $\psi / S^{n}: S^{n} \rightarrow S^{n}=i d / S^{n}$, it follows that $S^{n}$ is a retract of $P$. In particular, $P$ is not contractible.

When $X$ is diffeomorphic but different from $B^{n}$, the result is that $P$ has as a retract a diffeomorphic image of $S^{n}$, and is therefore not contractible.

Corollary 6. There exists no continuous aggregation rule $\phi:(P)^{m} \rightarrow P$ respecting anonymity and unanimity, for any $m \geqq 2$.

Proof. By Theorem 4, $P$ contains a continuous deformation of $S^{n}$ as a retract. Call this retract also $S^{n}$. Any continuous aggregation rule $\phi: P^{m} \rightarrow P$ which respects anonymity and unanimity would define another $\psi:\left(S^{n}\right)^{m} \rightarrow S^{n}$ 
with the same properties. However, the CW complex $S^{n}$ is not contractible. Therefore, by Theorem 1 of Chichilnisky and Heal (1983), $\psi$ cannot exist.

An alternative proof of this proposition is provided in Chichilnisky (1980).

\section{References}

Abraham, R. and J. Robbin, 1967, Transversal Mappings and Flows (W.A. Benjamin, New York). Chichilnisky, G., 1976, Manifolds of preferences and equilibria, Ph.D. dissertation (University of California, Berkeley, CA); Project on efficiency of decision making in economic systems, Technical report no. 27 (Harvard University, Cambridge, MA).

Chichilnisky, G., 1977a, Spaces of economic agents, Journal of Economic Theory.

Chichilnisky, G., 1977b, Nonlinear analysis and optimal economic growth, Journal of Mathematical Analysis and Applications.

Chichilnisky, G., 1980, Social choice and the topology of spaces of preferences, Advances in Mathematics.

Chichilnisky, G., 1981, Continuous representation of preferences, Review of Economic Studies.

Chichilnisky, G. and G. Heal, 1983, Necessary and sufficient conditions for the resolution of the social choice paradox, Journal of Economic Theory.

Debreu, G., 1964, Representation of a preference ordering by a numerical function, International Economic Review.

Debreu, G., 1972, Smooth preferences, Econometrica.

Hirsch, M., 1976, Differential Topology (Springer-Verlag, New York).

Hirsch, M. and B. Mazur, 1974, Smoothings of Piecewise Linear Manifolds, Annals of Mathematical Studies (Princeton University Press, Princeton, NJ).

Milnor, J., 1965, Topology from the Differentiable Viewpoint (The University Press of Virginia, Charlottesville, VA).

Skorohod, A.V., 1974, Integration in Hilbert Space (Springer-Verlag, New York).

Sobolev, S.L., 1963, Applications of Functional Analysis in Mathematical Physics, Translation of Math. Monographs, Vol. 7 (American Mathematical Society, Providence, RI).

Spanier, E.H., 1966, Algebraic Topology (McGraw-Hill, New York). 